\title{
EQUIVARIANT NORMAL FORM FOR NONDEGENERATE SINGULAR ORBITS OF INTEGRABLE HAMILTONIAN SYSTEMS
}

\author{
By EVA MIRANDA ${ }^{1}$ AND NGUYEN TIEN ZUNG
}

\begin{abstract}
We consider an integrable Hamiltonian system with $n$ degrees of freedom whose first integrals are invariant under the symplectic action of a compact Lie group $G$. We prove that the singular Lagrangian foliation associated to this Hamiltonian system is symplectically equivalent, in a $G$-equivariant way, to the linearized foliation in a neighborhood of a compact singular nondegenerate orbit. We also show that the nondegeneracy condition is not equivalent to the nonresonance condition for smooth systems.

(C) 2004 Elsevier SAS
\end{abstract}

RÉSUMÉ. - On considère un système hamiltonien intégrable à $n$ degrés de liberté et une action symplectique d'un groupe de Lie compact $G$ qui laisse invariantes les intégrales premières. On prouve que le feuilletage lagrangien singulier attaché à ce système hamiltonien est symplectiquement équivalent, de façon $G$-équivariante, au feuilletage linéarisé dans un voisinage d'une orbite compacte singulière. On démontre aussi que la condition de non-dégénérescence n'est pas équivalente à la non-résonance pour les systèmes différentiables.

() 2004 Elsevier SAS

\section{Introduction}

In this paper, we are interested in the geometry of integrable Hamiltonian systems on symplectic manifolds. When we refer to an integrable Hamiltonian system, we mean that it is integrable in the sense of Liouville. That is to say, the system is given by a moment map $\mathbf{F}$ on $\left(M^{2 n}, \omega\right)$,

$$
\mathbf{F}=\left(F_{1}, \ldots, F_{n}\right):\left(M^{2 n}, \omega\right) \rightarrow \mathbb{R}^{n}
$$

whose component functions $F_{i}$ are functionally independent almost everywhere and Poisson commuting $\left(\left\{F_{i}, F_{j}\right\}=0\right.$ for any $\left.i, j\right)$. The Hamiltonian system considered will be called smooth or real analytic if the corresponding moment map is so. Let $X=X_{H}$ be the Hamiltonian vector field associated to a given function $H$, we say that this Hamiltonian vector field is integrable if there exists a moment map with $F_{1}=H$.

In our approach to the study of the integrable Hamiltonian system, the original Hamiltonian function defining the system will be left aside and our study will be focused on the singular Lagrangian fibration given by the level sets of the moment map. This moment map generates an infinitesimal Poisson $\mathbb{R}^{n}$-action on $\left(M^{2 n}, \omega\right)$ via the Hamiltonian vector fields $X_{F_{1}}, \ldots, X_{F_{n}}$.

\footnotetext{
${ }^{1}$ Partially supported by the DGICYT project number BFM2003-03458. 
Denote by $O$ an orbit of this $\mathbb{R}^{n}$-action. We will assume that $O$ is a closed submanifold (i.e. compact without boundary) of $\left(M^{2 n}, \omega\right)$. Then it is well known that $O$ is diffeomorphic to a torus since the vector fields $X_{F_{1}}, \ldots, X_{F_{n}}$ are complete on $O$, and $O$ is a quotient of the Abelian group $\mathbb{R}^{n}$ by a cocompact subgroup.

The complete integrability of Hamiltonian systems is an old problem. In the XIX century, Joseph Liouville [13] proved that if a Hamiltonian system has $n$ functionally independent integrals in involution then it is integrable by quadratures. To our knowledge, Henri Mineur was the first who gave a complete description, up to symplectomorphism, of the Hamiltonian system in a neighborhood of a compact regular orbit of dimension $n$. In his papers [16-18], it is proven that under the previous assumptions, there is a symplectomorphism $\phi$ from a neighborhood $(\mathcal{U}(O), \omega)$ of $O$ in $\left(M^{2 n}, \omega\right)$ to $\left(D^{n} \times \mathbb{T}^{n}, \sum_{1}^{n} d \nu_{i} \wedge d \mu_{i}\right)$, where $\left(\nu_{1}, \ldots, \nu_{n}\right)$ is a coordinate system on a ball $D^{n}$, and $\left(\mu_{1}(\bmod 1), \ldots, \mu_{n}(\bmod 1)\right)$ is a periodic coordinate system on the torus $\mathbb{T}^{n}$, such that $\phi_{*} \mathbf{F}$ is a map which depends only on the variables $\nu_{1}, \ldots, \nu_{n}$. The functions $q_{i}=\phi^{*} \mu_{i}$ on $\mathcal{U}(O)$ are called angle variables, and the functions $p_{i}=\phi^{*} \nu_{i}$ are called action variables.

Although the works of Henri Mineur date back to the thirties, the theorem stated above has been known in the literature as Arnold-Liouville theorem.

Mineur $[16,18]$ also showed that the action functions $p_{i}$ can be defined via the period integrals:

$$
p_{i}(x)=\int_{\Gamma_{i}(x)} \beta .
$$

Here $\beta$ is a primitive 1 -form of the symplectic form, i.e. $d \beta=\omega$, and $\Gamma_{i}(x)$ for each point $x$ near $O$ is a closed curve which depends smoothly on $x$ and which lies on the Liouville torus containing $x$. The homology classes of $\Gamma_{1}(x), \ldots, \Gamma_{n}(x)$ form a basis of the first homology group of the Liouville torus. The above important formula for finding action functions will be called Mineur's formula. It can be used to find action functions and hence torus actions and normalization not only near regular level sets of the moment map, but also near singular level sets as well, see e.g. [26,27]. In particular, in [27] this Mineur's formula was used in the proof of the existence of a local analytic Birkhoff normalization for any analytic integrable Hamiltonian system near a singular point.

The above-mentioned action-angle coordinates entail a "uniqueness" for the symplectic structure and the regular Lagrangian fibration in a neighborhood of a compact orbit. In fact, they provide a "linear model" in a neighborhood of a regular compact orbit. In the same spirit, the aim of the present paper is to establish an analog of this classical Liouville-Mineur-Arnold theorem for the case when the orbit $O$ is singular, i.e. is of dimension $m=\operatorname{dim} O$ smaller than $n$, under a natural nondegeneracy condition. We will show that the system can be "linearized" near $O$ up to fibration-preserving symplectomorphisms. The fibration in question is the singular Lagrangian fibration given by the moment map. We also take into account the possible symmetries of the system. Namely, we will show that in the case there exists a symplectic action of a compact Lie group in a neighborhood of $O$ preserving the moment map, this linearization can be carried out in an equivariant way.

\section{Preliminaries and statement of the main results}

\subsection{Nondegenerate orbits}

In this paper, the orbit $O$ is assumed to be nondegenerate. The concept of nondegenerate orbit was introduced by Eliasson. Let us recall what it means (see, e.g., [9], [25]). A point $x \in M^{2 n}$ is 
called singular for the system if its rank, i.e. the rank of $\mathbf{F}$ at $x$, is smaller than $n$. If $\operatorname{rank} x=m$ then $m$ is also the dimension of the orbit through $x$ of the local Poisson $\mathbb{R}^{n}$ action. If rank $x=0$, i.e. $x$ is a fixed point, then the quadratic parts $F_{1}^{(2)}, \ldots, F_{n}^{(2)}$ of the components $F_{1}, \ldots, F_{n}$ of the moment map at $x$ are Poisson-commuting and they form an Abelian subalgebra, $\mathcal{A}$, of the Lie algebra $Q(2 n, \mathbb{R})$ of homogeneous quadratic functions of $2 n$ variables under the standard Poisson bracket. Observe that the algebra $Q(2 n, \mathbb{R})$ is isomorphic to the symplectic algebra $s p(2 n, \mathbb{R})$. A point $x$ will be called a nondegenerate fixed point if $\mathcal{A}$ is a Cartan subalgebra of $Q(2 n, \mathbb{R})$.

More generally, when $\operatorname{rank} x=m \geqslant 0$, we may assume without loss of generality that $d F_{1} \wedge \cdots \wedge d F_{m}(x) \neq 0$, and a local symplectic reduction near $x$ with respect to the local free $\mathbb{R}^{m}$-action generated by the Hamiltonian vector fields $X_{F_{1}}, \ldots, X_{F_{m}}$ will give us an $m$-dimensional family of local integrable Hamiltonian systems with $n-m$ degrees of freedom. Under this reduction, $x$ will be mapped to a fixed point in the reduced system, and if this fixed point is nondegenerate according to the above definition, then $x$ is called a nondegenerate singular point of rank $m$ and corank $(n-m)$. The orbit $O$ will be called nondegenerate if it contains a nondegenerate singular point. In fact, if a point in $O$ is nondegenerate then every point of $O$ is nondegenerate because nondegeneracy is a property which is invariant under the local Poisson $\mathbb{R}^{n}$-action.

\subsection{The Williamson type of an orbit}

According to [25] we will define the Williamson type of a nondegenerate singular point $x \in O$ as a triple of nonnegative integers $\left(k_{e}, k_{h}, k_{f}\right)$, where $k_{e}$ (resp., $\left.k_{h}, k_{f}\right)$ is the number of elliptic (resp., hyperbolic, focus-focus) components of the system at $x$. Let us recall what $k_{e}, k_{h}$ and $k_{f}$ stand for. When $\operatorname{rank} x=0$, a generic linear combination of the linear parts of the Hamiltonian vector fields $X_{F_{1}}, \ldots, X_{F_{n}}$ at $x$ has $k_{e}$ pairs of purely imaginary eigenvalues, $k_{h}$ pairs of real eigenvalues, and $k_{f}$ quadruples of nonreal nonpurely-imaginary complex eigenvalues (note that the set of eigenvalues is symmetric with respect to the real axis and the imaginary axis). If rank $x \neq 0$, we can perform a symplectic reduction first and the values of $k_{e}, k_{h}$ and $k_{f}$ coincide with the values of $k_{e}, k_{h}$ and $k_{f}$ at the point corresponding to $x$ in the reduced space. In particular, we have $k_{e}+k_{h}+2 k_{f}=n-m$. The triple $\left(k_{e}, k_{h}, k_{f}\right)$ is also called the Williamson type of $O$, because it does not depend on the choice of $x$ in $O$. When $k_{h}=k_{f}=0$, we say that the singular orbit is of elliptic type.

\subsection{The linear model}

We are going to introduce the linear model associated to the orbit $O$ for a given symplectic action preserving the system. Later, we will see that the invariants associated to the linear model are the Williamson type of the orbit and a twisting group $\Gamma$ attached to it.

Denote by $\left(p_{1}, \ldots, p_{m}\right)$ a linear coordinate system of a small ball $D^{m}$ of dimension $m$, $\left(q_{1}(\bmod 1), \ldots, q_{m}(\bmod 1)\right)$ a standard periodic coordinate system of the torus $\mathbb{T}^{m}$, and $\left(x_{1}, y_{1}, \ldots, x_{n-m}, y_{n-m}\right)$ a linear coordinate system of a small ball $D^{2(n-m)}$ of dimension $2(n-m)$. Consider the manifold

$$
V=D^{m} \times \mathbb{T}^{m} \times D^{2(n-m)}
$$

with the standard symplectic form $\sum d p_{i} \wedge d q_{i}+\sum d x_{j} \wedge d y_{j}$, and the following moment map:

$$
(\mathbf{p}, \mathbf{h})=\left(p_{1}, \ldots, p_{m}, h_{1}, \ldots, h_{n-m}\right): V \rightarrow \mathbb{R}^{n}
$$


where

$$
\begin{aligned}
& h_{i}=x_{i}^{2}+y_{i}^{2} \quad \text { for } 1 \leqslant i \leqslant k_{e}, \\
& h_{i}=x_{i} y_{i} \quad \text { for } k_{e}+1 \leqslant i \leqslant k_{e}+k_{h}, \\
& h_{i}=x_{i} y_{i+1}-x_{i+1} y_{i} \text { and } \\
& h_{i+1}=x_{i} y_{i}+x_{i+1} y_{i+1} \quad \text { for } i=k_{e}+k_{h}+2 j-1,1 \leqslant j \leqslant k_{f} .
\end{aligned}
$$

Let $\Gamma$ be a group with a symplectic action $\rho(\Gamma)$ on $V$, which preserves the moment map $(\mathbf{p}, \mathbf{h})$. We will say that the action of $\Gamma$ on $V$ is linear if it satisfies the following property:

$\Gamma$ acts on the product $V=D^{m} \times \mathbb{T}^{m} \times D^{2(n-m)}$ componentwise; the action of $\Gamma$ on $D^{m}$ is trivial, its action on $\mathbb{T}^{m}$ is by translations (with respect to the coordinate system $\left.\left(q_{1}, \ldots, q_{m}\right)\right)$, and its action on $D^{2(n-m)}$ is linear with respect to the coordinate system $\left(x_{1}, y_{1}, \ldots, x_{n-m}, y_{n-m}\right)$.

Suppose now that $\Gamma$ is a finite group with a free symplectic action $\rho(\Gamma)$ on $V$, which preserves the moment map and which is linear. Then we can form the quotient symplectic manifold $V / \Gamma$, with an integrable system on it given by the same moment map as above:

$$
(\mathbf{p}, \mathbf{h})=\left(p_{1}, \ldots, p_{m}, h_{1}, \ldots, h_{n-m}\right): V / \Gamma \rightarrow \mathbb{R}^{n} .
$$

The set $\left\{p_{i}=x_{i}=y_{i}=0\right\} \subset V / \Gamma$ is a compact orbit of Williamson type $\left(k_{e}, k_{f}, k_{h}\right)$ of the above system. We will call the above system on $V / \Gamma$, together with its associated singular Lagrangian fibration, the linear system (or linear model) of Williamson type $\left(k_{e}, k_{f}, k_{h}\right)$ and twisting group $\Gamma$ (or more precisely, twisting action $\rho(\Gamma)$ ). We will also say that it is a direct model if $\Gamma$ is trivial, and a twisted model if $\Gamma$ is nontrivial.

A symplectic action of a compact group $G$ on $V / \Gamma$ which preserves the moment map $\left(p_{1}, \ldots, p_{m}, h_{1}, \ldots, h_{n-m}\right)$ will be called linear if it comes from a linear symplectic action of $G$ on $V$ which commutes with the action of $\Gamma$. In our case, let $\mathcal{G}^{\prime}$ denote the group of linear symplectic maps which preserve the moment map then this group is abelian and therefore this last condition is automatically satisfied. In fact $\mathcal{G}^{\prime}$ is isomorphic to $\mathbb{T}^{m} \times G_{1} \times G_{2} \times G_{3}$ being $G_{1}$ the direct product of $k_{e}$ special orthogonal groups $S O(2, \mathbb{R}), G_{2}$ the direct product of $k_{h}$ components of type $S O(1,1, \mathbb{R})$ and $G_{3}$ the direct product of $k_{f}$ components of type $\mathbb{R} \times S O(2, \mathbb{R})$, respectively.

Now we can formulate our main result, which is the equivariant symplectic linearization theorem for compact nondegenerate singular orbits of integrable Hamiltonian systems:

THEOREM 2.1. - Under the above notations and assumptions, there exist a finite group $\Gamma, a$ linear system on the symplectic manifold $V / \Gamma$ given by (2.1)-(2.4), and a smooth Lagrangianfibration-preserving symplectomorphism $\phi$ from a neighborhood of $O$ into $V / \Gamma$, which sends $O$ to the torus $\left\{p_{i}=x_{i}=y_{i}=0\right\}$. The smooth symplectomorphism $\phi$ can be chosen so that via $\phi$, the system-preserving action of the compact group $G$ near $O$ becomes a linear system-preserving action of $G$ on $V / \Gamma$. If the moment map $\mathbf{F}$ is real analytic and the action of $G$ near $O$ is analytic, then the symplectomorphism $\phi$ can also be chosen to be real analytic. If the system depends smoothly (resp., analytically) on a local parameter (i.e. we have a local family of systems), then $\phi$ can also be chosen to depend smoothly (resp., analytically) on that parameter.

Remarks. -

(1) In the case when $O$ is a point and $G$ is trivial, the above theorem is due to Vey [22] in the analytic case, and Eliasson [8,9] in the smooth case. The smooth one-degree-of freedom case is due to Colin de Verdière and Vey [3]. 
(2) In the case when $O$ is of elliptic type and $G$ is trivial, the above theorem is due to Dufour and Molino [7] and Eliasson [9].

(3) The analytic case with $G$ trivial of the above theorem is due to Ito [12].

(4) The case with $n=2, m=1, O$ of hyperbolic type and $G$ trivial is due to Colin de Verdière and Vũ Ngọc San [4], and Currás-Bosch and the first author [6]. The symplectic uniqueness for the linear twisted hyperbolic case is not completely established in [6]. A complete proof for the twisted hyperbolic case when $n=2$ and $m=1$ was given by Currás-Bosch in [5]. The general smooth nonelliptic case seems to be new.

(5) A topological classification for nondegenerate singular fibers of the moment map (which contain nondegenerate singular orbits but are much more complicated in general) was obtained in [25], together with the existence of partial action-angle coordinate systems. However, the problem of classification up to symplectomorphisms of singular orbits or singular fibers was not considered in that paper.

(6) As it was already pointed out by Colin de Verdière and Vũ Ngọc San, the above theorem has direct applications in the problem of semiclassical quantization of integrable Hamiltonian systems. Of course, it is also useful for the global study of integrable Hamiltonian systems and their underlying symplectic manifolds.

(7) As it has been shown by the first author in [19], this theorem has applications in the analogous contact linearization problem for completely integrable systems on contact manifolds.

The rest of the paper is organized as follows: in Section 3 we study the case of a fixed point and give the corresponding $G$-equivariant result. As a by-product, we prove that the path component of the identity of the group of symplectomorphisms preserving the system is abelian. In Section 4 we prove the general case. In Appendix A we show that the nondegeneracy condition is not equivalent to the nonresonance condition for smooth systems.

\section{The case of a fixed point}

In this section we consider the case when $O$ is a point and we prove that the symplectic action of $G$ can be linearized symplectically in a fibration-preserving way.

This linearization result can be seen as a generalization of Bochner's linearization theorem [1] in the case the action of the group preserves additional structures: the symplectic form and the fibration. An equivariant Darboux theorem for symplectic actions of compact Lie groups in a neighborhood of a fixed point was proved by Weinstein in [24]. In the case the actions considered are the initial action and the linear action this equivariant Darboux theorem entails a symplectic linearization result in a neighbourhood of a fixed point (see for example [2] and [24]).

We will linearize the action of $G$ using the averaging method of Bochner's linearization. This averaging trick will be applied to fibration-preserving symplectomorphisms which will be presented as time-1-maps of Hamiltonian vector fields.

In order to linearize the action of the compact Lie group in a fibration preserving way we will work with a linear fibration and with the standard symplectic form. The results of Eliasson [8,9] (for smooth systems) and Vey [22] (for real analytic systems) show that there is a fibrationpreserving symplectomorphism from a neighborhood of $O$ in $\left(M^{2 n}, \omega, \mathbf{F}\right)$ to a neighborhood of the origin of the linear system $\left(\mathbb{R}^{2 n}, \sum_{i=1}^{n} d x_{i} \wedge d y_{i}, \mathbf{h}\right)$, where $\mathbf{h}=\left(h_{1}, \ldots, h_{n}\right)$ is the quadratic moment map given by formula (2.3). If the compact symmetry group $G$ in Theorem 2.1 is trivial, then we are done. Suppose now that $G$ is nontrivial. We can (and will) assume that the singular Lagrangian fibration near $O$ is already linear. We will refer to this Lagrangian fibration as $\mathcal{F}$. It remains to linearize the action of $G$ in such a way that the fibration remains the same. It would 
be interesting to adapt the proof of Eliasson for actions of compact Lie group but unfortunately some of the steps in his proof do not seem to admit an equivariant version.

Let us fix some notation that we will use throughout the paper. The vector field $X_{\Psi}$ will stand for a Hamiltonian vector field with associated Hamiltonian function $\Psi$. We denote by $\phi_{X}^{s}$ the time-s-map of the vector field $X$. Let $\psi$ be a local diffeomorphism $\psi:\left(\mathbb{R}^{2 n}, 0\right) \rightarrow\left(\mathbb{R}^{2 n}, 0\right)$. In the sequel, we will denote by $\psi^{(1)}$ the linear part of $\psi$ at 0 . That is to say, $\psi^{(1)}(x)=d_{0} \psi(x)$.

The group $\mathcal{G}$ stands for the group of local automorphisms preserving the system, $\mathcal{G}=$ $\left\{\phi:\left(\mathbb{R}^{2 n}, 0\right) \rightarrow\left(\mathbb{R}^{2 n}, 0\right)\right.$, such that $\left.\phi^{*}(\omega)=\omega, \mathbf{h} \circ \phi=\mathbf{h}\right\}$, and $\mathcal{G}_{0}$ stands for the path-component of the identity of $\mathcal{G}$. We denote by $\mathfrak{g}$ the Lie algebra of germs of Hamiltonian vector fields tangent to the fibration $\mathcal{F}$.

The subgroup of linear transformations contained in $\mathcal{G}$ is denoted by $\mathcal{G}^{\prime}$. As we have observed in the introduction $\mathcal{G}^{\prime}$ is abelian.

The goal of this section is to prove a local linearization result (Proposition 3.6) for a given smooth action of a compact Lie group $G$. In order to prove this result we will have to show that given any local automorphism $\psi \in \mathcal{G}$ then $\psi^{(1)} \circ \psi^{-1}$ can be presented as the time-1-flow of a Hamiltonian vector field as it is shown in Corollary 3.4.

As we will see this is, in fact, a consequence of Theorem 3.2 which shows that the exponential mapping exp : $\mathfrak{g} \rightarrow \mathcal{G}_{0}$ determined by the time-1-flow of a vector field $X \in \mathfrak{g}$ is a surjective group homomorphism.

Before stating this theorem we need the following sublemma.

Sublemma 3.1. - The Lie algebra $\mathfrak{g}$ is abelian and for any pair of vector fields $X_{G_{1}}$ and $X_{G_{2}}$ contained in $\mathfrak{g}$ the following formula holds

$$
\phi_{X_{G_{1}}+X_{G_{2}}}^{s}=\phi_{X_{G_{1}}}^{s} \circ \phi_{X_{G_{2}}}^{s} .
$$

Proof. - Let $X_{G_{1}}$ and $X_{G_{2}}$ be two vector fields in $\mathfrak{g}$. Since $\left\{G_{1}, G_{2}\right\}=\omega\left(X_{G_{1}}, X_{G_{2}}\right)$ and $X_{G_{1}}$ and $X_{G_{2}}$ are tangent to the Lagrangian fibration $\mathcal{F}$ then $\left\{G_{1}, G_{2}\right\}_{L}=0$ for any regular fiber $L$ of $\mathcal{F}$. On the other hand, since the set of regular fibers is dense and $X_{G_{1}}$ and $X_{G_{2}}$ are also tangent along the singular fibers, the bracket $\left\{G_{1}, G_{2}\right\}$ vanishes everywhere.

This implies in turn that $\left[X_{G_{1}}, X_{G_{2}}\right]=0$ and the Lie algebra $\mathfrak{g}$ is abelian. Therefore the flows associated to $X_{G_{1}}$ and $X_{G_{2}}$ commute. As a consequence $\alpha_{s}=\phi_{X_{G_{1}}}^{s} \circ \phi_{X_{G_{2}}}^{s}$ is a one-parameter subgroup.

A simple computation shows that its infinitesimal generator is $X_{G_{1}}+X_{G_{2}}$ and this ends the proof of the sublemma.

Observe that given a vector field $X_{G}$ in $\mathfrak{g}$, its time-s-flow $\phi_{X_{G}}^{s}$ preserves the moment map $\mathbf{h}$ because $X_{G}$ is tangent to $\mathcal{F}$. It also preserves the symplectic form since it is the flow of a Hamiltonian vector field. Finally since the vector field $X_{G}$ vanishes at the origin the mapping $\phi_{X_{G}}^{s}$ fixes the origin. Therefore, $\phi_{X_{G}}^{s}$ is contained in $\mathcal{G}$. In fact it is contained in $\mathcal{G}_{0}$ since $\phi_{X_{G}}^{0}=\mathrm{Id}$.

We denote by $\exp : \mathfrak{g} \rightarrow \mathcal{G}_{0}$ the exponential mapping defined by $\exp \left(X_{G}\right):=\phi_{X_{G}}^{1}$ for any $X_{G} \in \mathfrak{g}$. We can now state and prove the first theorem of this section.

THEOREM 3.2. - The exponential $\exp : \mathfrak{g} \rightarrow \mathcal{G}_{0}$ is a surjective group homomorphism, and moreover there is an explicit right inverse given by

$$
\phi \in \mathcal{G}_{0} \mapsto \int_{0}^{1} X_{t} d t \in \mathfrak{g},
$$


where $X_{t} \in \mathfrak{g}$ is defined by

$$
X_{t}\left(R_{t}\right)=\frac{d R_{t}}{d t}
$$

for any $C^{1}$ path $R_{t}$ contained in $\mathcal{G}_{0}$ connecting the identity to $\phi$.

Proof. - The formula proved in Sublemma 3.1 with $s=1$ shows that the exponential is a group homomorphism. It remains to show that it is surjective.

Let $R_{s}$ be a $C^{1}$ path in $\mathcal{G}_{0}$ such that $R_{0}=\mathrm{Id}$ and $R_{1}=\phi$ for a given $\phi \in \mathcal{G}_{0}$.

We define the time-dependent vector field $X_{t}$ by the following formula:

$$
X_{t}\left(R_{t}\right)=\frac{d R_{t}}{d t} .
$$

Observe that $X_{t}$ is tangent to the fibration $\mathcal{F}$ for any $t$ contained in $[0,1]$ because the diffeomorphism $R_{t}$ preserves the fibration $\mathcal{F}, \forall t$. On the other hand since $R_{t}$ is a symplectomorphism for any $t$, the vector field $X_{t}$ is locally Hamiltonian. Since the symplectic manifold considered is a neighbourhood $U$ of the origin the vector field is indeed Hamiltonian. Thus, $X_{t}$ is contained in the Lie algebra $\mathfrak{g}$.

Now consider $Y_{t}=\int_{0}^{t} X_{r} d r$. This vector field is also contained in $\mathfrak{g}$.

We will show that $\exp Y_{t}=R_{t}$ for any $t \in[0,1]$. Particularizing $t=1$, this shows that $\exp Y_{1}=R_{1}=\phi$ and therefore the mapping of the statement is an explicit right inverse of the exponential mapping and the exponential is surjective.

In order to show the equality $\exp Y_{t}=R_{t}, t \in[0,1]$. We will show that it satisfies the same nonautonomous differential equation

$$
X_{t}\left(\exp Y_{t}\right)=\frac{d\left(\exp Y_{t}\right)}{d t}
$$

and this will imply $\exp Y_{t}=R_{t}, t \in[0,1]$, since the initial conditions are the same.

In fact, we will prove that

$$
s X_{t}\left(\phi_{Y_{t}}^{s}\right)=\frac{d \phi_{Y_{t}}^{s}}{d t}
$$

which leads to the desired result when $s=1$ since by definition $\phi_{Y_{t}}^{1}=\exp Y_{t}$.

Observe that the formula we want to prove is equivalent to the fact that the vector field $s X_{t}$ is tangent to the curve $\phi_{Y_{t+u}}^{s} \circ\left(\phi_{Y_{t}}^{s}\right)^{-1}$ at any point $p$. Therefore, we can write formula (3.1) as

$$
s X_{t}=\left.\frac{d}{d u}\right|_{\mid u=0}\left(\phi_{Y_{t+u}}^{s}\left(\phi_{Y_{t}}^{s}\right)^{-1}\right) .
$$

After differentiation with respect to $s$ the formula we want to prove becomes:

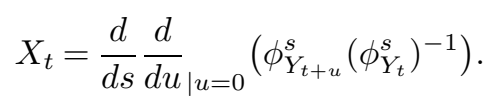

We will first compute

$$
\frac{d}{d s}\left(\phi_{Y_{t+u}}^{s}\left(\phi_{Y_{t}}^{s}\right)^{-1}\right)
$$

According to Sublemma 3.1 the Lie algebra $\mathfrak{g}$ is abelian and we may write

$$
\left(\phi_{Y_{t+u}}^{s}\left(\phi_{Y_{t}}^{s}\right)^{-1}\right)=\phi_{Y_{t+u}-Y_{t}}^{s}
$$


Observe that $Y_{t+u}-Y_{t}=\int_{t}^{t+u} X_{r} d r$.

On the one hand using the definition of flow

$$
\frac{d}{d s}\left(\phi_{Y_{t+u}}^{s}\left(\phi_{Y_{t}}^{s}\right)^{-1}\right)=\frac{d}{d s}\left(\phi_{\int_{t}^{t+u} X_{r} d r}\right)=\int_{t}^{t+u} X_{r} d r
$$

on the other hand, since

$$
\lim _{u \rightarrow 0} \frac{\int_{t}^{t+u} X_{r} d r-u X_{t}}{u}=0,
$$

we can write $\int_{t}^{t+u} X_{r} d r=u X_{t}+\mathrm{o}(u)$, uniformly in $t$.

Therefore,

$$
\frac{d}{d s}\left(\phi_{Y_{t+u}}^{s}\left(\phi_{Y_{t}}^{s}\right)^{-1}\right)=u X_{t}+\mathrm{o}(u) .
$$

Finally differentiating in $u$ and particularizing $u=0$ we obtain

$$
\left.\frac{d}{d u} \frac{d}{d s}\right|_{\mid u=0}\left(\phi_{Y_{t+u}}^{s}\left(\phi_{Y_{t}}^{s}\right)^{-1}\right)_{\mid u=0}=X_{t}
$$

This proves formula (3.2) and this ends the proof of the theorem.

Remark. - Observe that this exponential mapping is not always injective. Since a vector field $X \in \mathfrak{g}$ is a Hamiltonian vector field tangent to $\mathcal{F}$, its Hamiltonian function is a first integral of the system given by $\mathbf{h}$. Therefore $X=X_{\phi\left(h_{1}, \ldots, h_{n}\right)}$ when restricted to each connected component of the regular set of $\mathbf{h}$. Bearing this in mind, it is easy to see that the exponential is injective if there are only hyperbolic components (Williamson type $(0, n, 0)$ ). If there are elliptic or focus-focus components any vector field of type $X=X_{2 \pi k h_{i}}, k \in \mathbb{Z}$ (with $h_{i}$ standing for an elliptic function or for a function $h_{i}$ in a focus-focus pair $h_{i}, h_{i+1}$ ) is contained in the kernel of the exponential. In fact, the kernel is generated by these vector fields. In particular, this guarantees that exp is always locally injective.

The theorem above has direct applications to the linearization problem posed at the beginning of this section but it also tells us that $\mathcal{G}_{0}$ is abelian.

\section{COROLlary 3.3. - The group $\mathcal{G}_{0}$ is abelian.}

Proof. - According to Theorem 3.2, the exponential mapping is a surjective morphism of groups and according to Sublemma 3.1 the Lie algebra $\mathfrak{g}$ is abelian. This implies that $\mathcal{G}_{0}$ is abelian.

\section{Remarks. -}

(1) One could also check that $\mathcal{G}_{0}$ is abelian using the following: Observe that it is enough to check that any two diffeomorphisms $\phi_{1}$ and $\phi_{2}$ in $\mathcal{G}_{0}$ commute on an open dense set. We consider the dense set $\Omega$ determined by the regular points of the fibration. Now consider the submanifold $L_{\left(\delta_{1}, \ldots, \delta_{n}\right)}=\left\{\left(x_{1}, y_{1}, \ldots, x_{n}, y_{n}\right), x_{i}=\delta_{i} y_{i}, \forall i\right\}$ with $\delta_{i} \in\{-1,+1\}$. It is a Lagrangian submanifold. In the case there are no hyperbolic components, taking $\delta_{i}=1$ and $\delta_{i+1}=-1$ for the focus-focus pairs $h_{i}$ and $h_{i+1}$, the submanifold $L_{\left(\delta_{1}, \ldots, \delta_{n}\right)}$ is transversal to the regular Lagrangian fibration induced by $\mathbf{F}$ on $\Omega$. So we may apply a result of Weinstein [23] which ensures that the foliation is symplectomorphic in a neighborhood of $L$ to the foliation by fibers in $T^{*}(L)$ endowed 
with the Liouville symplectic structure. In this way we may assume that the symplectic form is $\omega=\sum_{i} d p_{i} \wedge d q_{i}$ being $q_{i}$ coordinates on $L$. The fibration is then determined by $\mathbf{F}=\left(q_{1}, \ldots, q_{n}\right)$. Now any diffeomorphism lying in $\mathcal{G}$ can be written in the form $\phi\left(q_{1}, \ldots, q_{n}, p_{1}, \ldots, p_{n}\right)=\left(q_{1}, \ldots, q_{n}, p_{1}+\alpha_{1}(q), \ldots, p_{n}+\alpha_{n}(q)\right)$ for certain smooth functions $\alpha_{i}$. Clearly any two diffeomorphisms of this form commute and therefore this proves that $\mathcal{G}_{0}$ is abelian. In the case there are hyperbolic components since $\phi_{1}$ and $\phi_{2}$ leave each orthant invariant. In each orthant we may consider an appropriate choice of $\delta_{i}$ for hyperbolic functions $h_{i}$ such that $L_{\left(\delta_{1}, \ldots, \delta_{n}\right)}$ is a transversal Lagrangian submanifold to the fibration restricted to this orthant. And we may repeat the argument above for $F$ restricted to each orthant to conclude that $\mathcal{G}_{0}$ is abelian.

As observed by Weinstein in [23], the study of local symplectomorphism preserving the foliation by fibers in $T^{*}(L)$ has relevance in the study of lagrangian-foliated symplectic manifolds.

(2) Although $\mathcal{G}$ is also abelian for analytical systems, it is not always abelian if we consider smooth systems as the following example shows:

Consider $n=1$ and $h=x y$. Let $\psi$ be the smooth function:

$$
\psi(x, y)= \begin{cases}\mathrm{e}^{-1 /(x y)^{2}}, & x \geqslant 0, \\ 2 \mathrm{e}^{-1 /(x y)^{2}}, & x \leqslant 0,\end{cases}
$$

and let $\phi$ be the time-1-map of $X_{\psi}$. Then $\phi$ does not commute with the involution $I(x, y)=(-x,-y)$.

Another interesting consequence of Theorem 3.2 is the following result about the local automorphisms of the linear integrable system $\left(\mathbb{R}^{2 n}, \sum_{i=1}^{n} d x_{i} \wedge d y_{i}, \mathbf{h}\right)$.

COROllaRY 3.4. - Suppose that $\psi:\left(\mathbb{R}^{2 n}, 0\right) \rightarrow\left(\mathbb{R}^{2 n}, 0\right)$ is a local symplectic diffeomorphism of $\mathbb{R}^{2 n}$ which preserves the quadratic moment map $\mathbf{h}=\left(h_{1}, \ldots, h_{n}\right)$. Then,

(1) The linear part $\psi^{(1)}$ is also a system-preserving symplectomorphism.

(2) There is a vector field contained in $\mathfrak{g}$ such that its time-1-map is $\psi^{(1)} \circ \psi^{-1}$. Moreover, for each vector field $X$ fulfilling this condition there is a unique local smooth function $\Psi:\left(\mathbb{R}^{2 n}, 0\right) \rightarrow \mathbb{R}$ vanishing at 0 which is a first integral for the linear system given by $\mathbf{h}$ and such that $X=X_{\Psi}$. If $\psi$ is real analytic then $\Psi$ is also real analytic.

Proof. - We are going to construct a path connecting $\psi$ to $\psi^{(1)}$ contained in

$$
\mathcal{G}=\left\{\phi:\left(\mathbb{R}^{2 n}, 0\right) \rightarrow\left(\mathbb{R}^{2 n}, 0\right), \text { such that } \phi^{*}(\omega)=\omega, \mathbf{h} \circ \phi=\mathbf{h}\right\} .
$$

Given a map $\psi \in \mathcal{G}$, we consider

$$
S_{t}^{\psi}(x)= \begin{cases}\frac{\psi \circ g_{t}}{t}(x), & t \in(0,2], \\ \psi^{(1)}(x), & t=0,\end{cases}
$$

being $g_{t}$ the homothety $g_{t}\left(x_{1}, \ldots, x_{n}\right)=t\left(x_{1}, \ldots, x_{n}\right)$.

Observe that in case $\psi$ is smooth, this mapping $S_{t}^{\psi}$ is smooth and depends smoothly on $t$. In case $\psi$ is real analytic, the corresponding $S_{t}^{\psi}$ is also real analytic and depends analytically on $t$.

First let us check that $h \circ S_{t}^{\psi}=h$ when $t \neq 0$. We do it component-wise. 
Let $x=\left(x_{1}, \ldots, x_{n}\right)$, then

$$
h_{j} \circ\left(\frac{\psi \circ g_{t}}{t}\right)(x)=\frac{\left(h_{j} \circ \psi \circ g_{t}\right)(x)}{t^{2}}=\frac{h_{j} \circ g_{t}(x)}{t^{2}}=h_{j}(x),
$$

where in the first and the last equalities we have used the fact that each component $h_{j}$ of the moment map $h$ is a quadratic polynomial whereas the condition $h \circ \psi=h$ yields the second equality.

Now we check that $\left(S_{t}^{\psi}\right)^{*}(\omega)=\omega$ when $t \neq 0$. Since $\omega=\sum d x_{i} \wedge d y_{i}$, then $g_{t}^{*}(\omega)=t^{2} \omega$. But since $\psi$ preserves $\omega$ then

$$
\left(S_{t}^{\psi}\right)^{*}(\omega)=\left(\frac{\psi \circ g_{t}}{t}\right)^{*} \omega=\omega
$$

when $t \neq 0$.

So far we have checked the conditions $h \circ S_{t}^{\psi}=h$ and $\left(S_{t}^{\psi}\right)^{*}(\omega)=\omega$ when $t \neq 0$ but since $S_{t}^{\psi}$ depends smoothly on $t$ we also have that $h \circ S_{0}^{\psi}=h$ and $\left(S_{0}^{\psi}\right)^{*}(\omega)=\omega$. So, in particular, we obtain that $S_{0}^{\psi}=\psi^{(1)}$ preserves the moment map and the symplectic structure and therefore $\psi^{(1)}$ is also contained in $\mathcal{G}$. This proves the first statement of the corollary.

In order to prove the second statement we only need to show that $\psi^{(1)} \circ \psi^{-1}$ is contained in $\mathcal{G}_{0}$. Then, we can apply Theorem 3.2 to conclude.

Consider

$$
R_{t}=\psi^{(1)} \circ S_{t}^{\left(\psi^{-1}\right)}
$$

with $t \in[0,1]$, this path connects the identity to $\psi^{(1)} \circ \psi^{-1}$ and is contained in $\mathcal{G}_{0}$.

Then the formula of Theorem 3.2 applied to this path provides a vector field $X$ whose time1-map is $\psi^{(1)} \circ \psi^{-1}$ and there exists a unique Hamiltonian function $\Psi$ vanishing at 0 such that $X_{\Psi}=X$. Since the vector field $X_{\Psi}$ is tangent to the foliation then $\left\{\Psi, h_{i}\right\}=0, \forall i$, in other words, $\Psi$ is a first integral of the system.

In the case the action of $G$ depends on parameters we have a parametric version of Corollary 3.4.

Corollary 3.5. - Let $D_{p}$ stand for a disk centered at 0 in the parameters $p_{1}, \ldots, p_{m}$. We denote by $\mathbf{p}=\left(p_{1}, \ldots, p_{m}\right)$. Assume that $\psi_{\mathbf{p}}:\left(\mathbb{R}^{2 n}, 0\right) \rightarrow\left(\mathbb{R}^{2 n}, 0\right)$ is a local symplectic diffeomorphism of $\mathbb{R}^{2 n}$ which preserves the quadratic moment map $\mathbf{h}$ and which depends smoothly on the parameters $\mathbf{p}$. Then there is a local smooth function $\Psi_{\mathbf{p}}:\left(\mathbb{R}^{2 n}, 0\right) \rightarrow \mathbb{R}$ vanishing at 0 depending smoothly on $\mathbf{p}$ which is a first integral for the linear system given by $\mathbf{h}$ and such that $\psi_{0}^{(1)} \circ \psi_{\mathbf{p}}^{-1}$ is the time-1 map of the Hamiltonian vector field $X_{\Psi_{\mathbf{p}}}$ of $\Psi_{\mathbf{p}}$. If $\psi_{\mathbf{p}}$ is real analytic and depends analytically on the parameters then $\Psi_{\mathrm{p}}$ is also real analytic and depends analytically on the parameters.

Proof. - We will apply again Theorem 3.2. We consider the path

$$
M_{t}=\psi_{0}^{(1)} \circ\left(\psi_{g_{t}(\mathbf{p})}\right)^{-1}
$$

where $g_{t}(p)=\left(t p_{1}, \ldots, t p_{m}\right)$. This path is smooth (resp. analytic) if $\psi$ is smooth (resp. analytic) and depends analytically on $t$ and is contained in $\mathcal{G}_{0}$. Because of Theorem 3.2 we can associate a Hamiltonian vector field $X_{\Psi_{\mathbf{p}}}$ to this path such that its time-1-map coincides with $\psi_{0}^{(1)} \circ\left(\psi_{\mathbf{p}}\right)^{-1}$. By construction, this function $\Psi_{\mathbf{p}}$ is smooth (or real analytic) if $\psi_{\mathbf{p}}$ is smooth (or real analytic) and depends smoothly or analytically on the parameters if $\psi_{\mathbf{p}}$ does so. 
After this digression, we will prove our local linearization result. By abuse of language, we will denote the local (a priori nonlinear) action of our compact group $G$ on $\left(\mathbb{R}^{2 n}, \sum_{i=1}^{n} d x_{i} \wedge d y_{i}, \mathbf{h}\right)$ by $\rho$. For each element $g \in G$, denote by $X_{\Psi(g)}$ the Hamiltonian vector field constructed via the formula explicited in Theorem 3.2 applied to the path $R_{t}$ explicited in Corollary 3.4. The time-1 map of this vector field is $\rho(g)^{(1)} \circ \rho(g)^{-1}$ where $\rho(g)^{(1)}$ denotes the linear part of $\rho(g)$. It is clear that this defines a smooth function $\Psi(g)$.

Consider the averaging of the family of vector fields $X_{\Psi(g)}$ over $G$ with respect to the normalized Haar measure $d \mu$ on $G$. That is to say

$$
X_{G}(x)=\int_{G} X_{\Psi(g)}(x) d \mu, \quad x \in \mathbb{R}^{2 n} .
$$

This vector field is Hamiltonian with Hamiltonian function $\int_{G} \Psi(g) d \mu$. Denote by $\Phi_{G}$ the time-1 map of this vector field $X_{G}$.

Proposition 3.6. $-\Phi_{G}$ is a local symplectic coordinate transformation of $\mathbb{R}^{2 n}$ which preserves the system $\left(\mathbb{R}^{2 n}, \sum_{i=1}^{n} d x_{i} \wedge d y_{i}, \mathbf{h}\right)$ and under which the action of $G$ becomes linear.

Proof. - Since $\Phi_{G}$ is the time-1 map of vector field contained in $\mathfrak{g}, \Phi_{G}$ is a symplectomorphism preserving the fibration. Therefore it defines a local symplectic variable transformation. Let us check that this transformation linearizes the action of $G$.

From the definition of $\Phi_{G}$ and formula (3.5),

$$
\Phi_{G}(x)=\phi_{X_{G}}^{1}(x)=\int_{G} \phi_{X_{\Psi(g)}}^{1}(x) d \mu .
$$

But since, $\phi_{X_{\Psi(g)}}^{1}=\rho(g)^{(1)} \circ \rho(g)^{-1}$ we have

$$
\Phi_{G}(x)=\int_{G} \rho(g)^{(1)} \circ \rho(g)^{-1}(x) d \mu .
$$

We proceed as in the proof of Bochner's linearization theorem [1]

$$
\left(\rho(h)^{(1)} \circ \Phi_{G} \circ \rho(h)^{-1}\right)(x)=\rho(h)^{(1)} \circ \int_{G}\left(\rho(g)^{(1)} \circ \rho(g)^{-1}\right)\left(\rho(h)^{-1}(x)\right) d \mu .
$$

Using the linearity of $\rho(h)^{(1)}$ and the fact that $\rho$ stands for an action, the expression above can be written as

$$
\int_{G}(\rho(h) \circ \rho(g))^{(1)} \circ(\rho(h) \circ \rho(g))^{-1}(x) d \mu .
$$

Finally this expression equals $\Phi_{G}$ due to the left invariance property of averaging and we have proven $\Phi_{G} \circ \rho(h)=\rho(h)^{(1)} \circ \Phi_{G}$ as we wanted.

Remark. - A fortiori, one can show that any analytic action of a compact Lie group $G$ on $\left(\mathbb{R}^{2 n}, \sum_{i=1}^{n} d x_{i} \wedge d y_{i}, \mathbf{h}\right)$ must be linear (so no need to linearize). Only in the smooth nonelliptic case the action of $G$ may be nonlinear. And even in the smooth case, if $G$ is connected then its action is also automatically linear. 
In the case the action of $G$ depends on parameters, this proposition and Corollary 3.4 lead to its parametric version.

PROPOSITION 3.7. - In the case the action $\rho_{\mathbf{p}}$ depends smoothly (resp. analytically) on parameters there exists a local symplectic coordinate transformation of $\mathbb{R}^{2 n}, \Phi_{\mathbf{p}}$, which preserves the system and which satisfies

$$
\Phi_{\mathbf{p}} \circ \rho_{\mathbf{p}}(h)=\rho_{0}(h)^{(1)} \circ \Phi_{\mathbf{p}} .
$$

The proposition above will be a key point in the proof of the linearization in a neighborhood of the orbit.

Remark.-In this section we have addressed a linearization problem with a foliation determined by a moment map $\mathbf{h}=\left(h_{1}, \ldots, h_{n}\right)$ corresponding to a nondegenerate singularity. Thanks to the smooth linearization result of Eliasson this moment map has very specific component functions $h_{i}$ of elliptic, hyperbolic and focus-focus type.

However, some of the results in this section do no use this particularity and remain valid in a more general context. For instance Sublemma 3.1 and Theorem 3.2 hold for a completely integrable system which defines a generically Lagrangian foliation and has the origin as singular point.

Corollaries 3.4 and 3.5 also remain valid if we also assume that the component functions $h_{i}$ are homogeneous because the path $S_{t}$ also preserves $\mathbf{h}$ under this condition. In particular, the final linearization results Propositions 3.6 and 3.7 also hold for foliations whose moment map has homogeneous component functions.

\section{The general case}

Suppose now that $\operatorname{dim} O=m>0$. For the moment, we will forget about the group $G$, and try to linearize the system in a nonequivariant way first.

First let us recall the following theorem proved by the second author in [25]:

THEOREM 4.1. - Let $(U(N), \mathcal{F})$ be a nondegenerate singularity of Williamson type $\left(k_{e}, k_{h}\right.$, $k_{f}$ ) of an integrable Hamiltonian system with $n$ degrees of freedom. Then there is a natural Hamiltonian action of a torus $\mathbb{T}^{n-k_{h}-k_{f}}$ which preserves the moment map of the integrable Hamiltonian system. This action is unique, up to automorphism of $\mathbb{T}^{n-k_{h}-k_{f}}$ and it is free almost everywhere in $U(N)$.

In this theorem $U(N)$ stands for a neighborhood of a leaf $N$. If we consider an orbit instead of a leaf of rank $m$ and Williamson type $\left(k_{e}, k_{h}, k_{f}\right)$ we obtain a locally free system-preserving torus $\mathbb{T}^{m}$-action in a neighborhood of the orbit $O$. In fact, this action can be found by using either Mineur's formula (1.2) or alternatively the flat affine structure on the (local) regular orbits near $O$ of the Poisson $\mathbb{R}^{n}$-action, and the existence of $m$ nonvanishing cycles on these orbits. Let us denote by $\left(p_{1}, \ldots, p_{m}\right)$ an $m$-tuple of action functions near $O$ which generates such a locally-free $\mathbb{T}^{m}$-action, and denote by $X_{1}, \ldots, X_{m}$ the $m$ corresponding periodic (of period 1 ) Hamiltonian vector fields.

Of course, $O$ is an orbit of the above $\mathbb{T}^{m}$-action. Denote by $\Gamma$ the isotropy group of the action of $\mathbb{T}^{m}$ on $O$. So $\Gamma$ is a finite abelian group. There is a normal finite covering $\widetilde{\mathcal{U}(O)}$ of a tubular neighborhood $\mathcal{U}(O)$ of $O$ such that the $\mathbb{T}^{m}$-action on $\mathcal{U}(O)$ can be pulled back to a free $\mathbb{T}^{m}$-action on $\widetilde{\mathcal{U}(O)}$. The symplectic form $\omega$, the moment map $\mathbf{F}$ and its corresponding singular Lagrangian fibration, and the action functions $p_{1}, \ldots, p_{m}$ can be pulled back to $\widetilde{\mathcal{U}(O)}$. 
We will use $\sim$ to denote the pull-back: for example, the pull-back of $O$ is denoted by $\widetilde{O}$, and the pull-back of $p_{1}$ is denoted by $\widetilde{p_{1}}$. The free action of $\Gamma$ on $\widetilde{\mathcal{U}(O)}$ commutes with the free $\mathbb{T}^{m}$-action. By cancelling out the translations (symplectomorphisms given by the $\mathbb{T}^{m}$-action are called translations), we get another action of $\Gamma$ on $\widetilde{\mathcal{U}(O)}$ which fixes $O$. We will denote this latter action by $\rho^{\prime}$.

Take a point $\widetilde{x} \in \widetilde{O}$, a local disk $\widetilde{P}$ of dimension $(2 n-m)$ which intersects $\widetilde{O}$ transversally at $\widetilde{x}$ and which is preserved by $\rho^{\prime}$. Denote by $\hat{q}_{1}, \ldots, \hat{q}_{m}$ the uniquely defined functions modulo 1 on $\widetilde{\mathcal{U}(O)}$ which vanish on $\widetilde{P}$ and such that $\widetilde{X}_{i}\left(q_{i}\right)=1, \widetilde{X_{i}}\left(q_{j}\right)=0$ if $i \neq j$. Then each local disk $\left\{\widetilde{p_{1}}=\right.$ const $, \ldots, \widetilde{p_{m}}=$ const $\} \cap \widetilde{P}$ near $\widetilde{x}$ has an induced symplectic structure, induced singular Lagrangian fibration of an integrable Hamiltonian system with a nondegenerate fixed point, which is invariant under the action $\rho^{\prime}$ of $\Gamma$. Applying the result of the previous section, i.e. Theorem 2.1 in the case with a fixed point, compact symmetry group $\Gamma$, and parameters $p_{1}, \ldots, p_{m}$, we can define local functions $\widetilde{x_{1}}, \widetilde{y_{1}}, \ldots, \widetilde{x_{n-m}}, \widetilde{y_{n-m}}$ on $\widetilde{P}$, such that they form a local symplectic coordinate system on each local disk $\left\{\widetilde{p_{1}}=\right.$ const, $\ldots, \widetilde{p_{m}}=$ const $\} \cap \widetilde{P}$, with respect to which the induced Lagrangian fibration is linear and the action $\rho^{\prime}$ of $\Gamma$ is linear. We extend $\widetilde{x_{1}}, \widetilde{y_{1}}, \ldots, \widetilde{x_{n-m}}, \widetilde{y_{n-m}}$ to functions on $\widetilde{\mathcal{U}(O)}$ by making them invariant under the action of $\mathbb{T}^{m}$. Define the following symplectic form on $\widetilde{\mathcal{U}(O)}$ :

$$
\widetilde{\omega_{1}}=\sum \widetilde{d p_{i}} \wedge d \widetilde{q}_{i}+\sum d \widetilde{x_{i}} \wedge d \widetilde{y_{i}}
$$

Consider the difference between $\widetilde{\omega}$ and $\widetilde{\omega_{1}}$ :

LEMMA 4.2. - There exist functions $\widetilde{g}_{i}$ in a neighborhood of $\widetilde{O}$ in $\widetilde{\mathcal{U}(O)}$, which are invariant under the $\mathbb{T}^{m}$-action, and such that

$$
\widetilde{\omega_{1}}-\widetilde{\omega}=\sum d \widetilde{p_{i}} \wedge d \widetilde{g}_{i}
$$

Proof.-By definition of $\widetilde{\omega_{1}}$, the vector field $\widetilde{X_{i}}$ is also the Hamiltonian vector field of $\widetilde{p_{i}}$ with respect to $\widetilde{\omega_{1}}$. Thus we have $\left.\widetilde{X_{i}}\right\lrcorner\left(\widetilde{\omega_{1}}-\widetilde{\omega}\right)=0$ and $\left.\widetilde{X_{i}}\right\lrcorner d\left(\widetilde{\omega_{1}}-\widetilde{\omega}\right)=0$. In other words, $\widetilde{\omega_{1}}-\widetilde{\omega}$ is a basic 2 -form with respect to the fibration given by the orbits of the $\mathbb{T}^{m}$-action, i.e. it can be viewed as a 2 -form on the $(2 n-m)$-dimensional space of variables $\left(\widetilde{p_{1}}, \ldots, \widetilde{p_{m}}, \widetilde{x_{1}}, \widetilde{y_{1}}, \ldots, \widetilde{x_{n-m}}, \widetilde{y_{n-m}}\right)$. Moreover, by construction, the restriction of $\widetilde{\omega_{1}}$ on each subspace $\left\{\widetilde{p_{1}}=\right.$ const $, \ldots, \widetilde{p_{m}}=$ const $\}$ coincides with the restriction of $\widetilde{\omega}$ on that subspace. Thus we can write

$$
\widetilde{\omega_{1}}-\widetilde{\omega}=\sum d \widetilde{p_{i}} \wedge \widetilde{\alpha}_{i}
$$

where each $\widetilde{\alpha_{i}}$ is an 1 -form in variables $\left(\widetilde{p_{1}}, \ldots, \widetilde{p_{m}}, \widetilde{x_{1}}, \widetilde{y_{1}}, \ldots, \widetilde{x_{n-m}}, \widetilde{y_{n-m}}\right)$. We have that $\sum d \widetilde{p_{i}} \wedge d \widetilde{\alpha_{i}}=d \widetilde{\omega_{1}}-d \widetilde{\omega}=0$, which implies that the restriction of $\widetilde{\alpha_{i}}$ on each subspace $\left\{\widetilde{p_{1}}=\right.$ const $, \ldots, \widetilde{p_{m}}=$ const $\}$ is closed (hence exact), so we can write $\widetilde{\alpha_{i}}$ as

$$
\widetilde{\alpha_{i}}=d \widetilde{\beta}_{i}+\sum \widetilde{c_{i j}} d \widetilde{p_{j}}
$$

where $\widetilde{\beta}_{i}$ and $\widetilde{c_{i j}}$ are functions of variables $\left(\widetilde{p_{1}}, \ldots, \widetilde{p_{m}}, \widetilde{x_{1}}, \widetilde{y_{1}}, \ldots, \widetilde{x_{n-m}}, \widetilde{y_{n-m}}\right)$. Thus

$$
\widetilde{\omega_{1}}-\widetilde{\omega}=\sum_{i<j}\left(\widetilde{c_{i j}}-\widetilde{c_{j i}}\right) d \widetilde{p_{i}} \wedge d \widetilde{p_{j}}+\sum d \widetilde{p_{i}} \wedge d \widetilde{\beta_{i}}
$$


Since $d\left(\widetilde{\omega_{1}}-\widetilde{\omega}\right)=0$, the 2-form $\sum_{i<j}\left(\widetilde{c_{i j}}-\widetilde{c_{j i}}\right) d \widetilde{p_{i}} \wedge d \widetilde{p_{j}}$ is closed (hence exact), and the functions $\widetilde{c_{i j}}-\widetilde{c_{j i}}$ are independent of the variables $\left(\widetilde{x_{1}}, \widetilde{y_{1}}, \ldots, \widetilde{x_{n-m}}, \widetilde{y_{n-m}}\right)$. Thus we can write

$$
\sum_{i<j}\left(\widetilde{c_{i j}}-\widetilde{c_{j i}}\right) d \widetilde{p_{i}} \wedge d \widetilde{p_{j}}=d\left(\sum d \widetilde{p_{i}} \wedge \widetilde{\gamma_{i}}\right)
$$

where $\widetilde{\gamma_{i}}$ are functions of variables $\left(\widetilde{p_{1}}, \ldots, \widetilde{p_{m}}\right)$. Now put $\widetilde{g}_{i}=\widetilde{\beta}_{i}+\widetilde{\gamma_{i}}$.

Consider the $\widetilde{g}_{i}$ given by the lemma and define

$$
\tilde{q}_{i}=\hat{q}_{i}-\widetilde{g}_{i}
$$

Then with respect to the coordinate system $\left(\widetilde{p_{i}}, \widetilde{q_{i}}, \widetilde{x_{j}}, \widetilde{y_{j}}\right)$, the symplectic form $\widetilde{\omega}$ has the standard form, the singular Lagrangian fibration is linear, and the free action of $\Gamma$ is also linear.

Remark. - There is another proof of Theorem 2.1 in the general case with trivial $G$ which does not use Lemma 4.2. It goes as follows. Assume that we have constructed the system of coordinates

$$
\widetilde{p}_{1}, \hat{q}_{1}, \widetilde{x}_{1}, \widetilde{y}_{1}, \ldots, \widetilde{p}_{m}, \hat{q}_{m} \widetilde{x}_{n-m}, \widetilde{y}_{n-m}
$$

as before. Let $\mathcal{D}_{R}$ be the symplectic distribution

$$
\mathcal{D}_{R}=\left\langle\frac{\partial}{\partial \widetilde{p}_{1}}, X_{1}, \ldots, \frac{\partial}{\partial \widetilde{p}_{m}}, X_{m}\right\rangle
$$

and let $\mathcal{D}_{S}$ be the distribution symplectically orthogonal to $\mathcal{D}_{R}$. Thus, we can write

$$
\widetilde{w}=\widetilde{\omega}_{R}+\widetilde{\omega}_{S} .
$$

It is easy to check that $D_{S}$ is an involutive distribution. Denote by $\mathcal{N}_{S}^{p}$ the integral manifold of $D_{S}$ through the point $p$ then $\widetilde{\omega}_{S}\left|p=\widetilde{\omega}_{\mathcal{N}_{S}^{p}}\right| p$. We can apply Theorem 2.1 to each $\mathcal{N}_{S}^{p}$ to obtain a new system of coordinates $\widehat{x_{1}}, \widehat{y_{1}}, \ldots, \widehat{x_{n-m}}, \widehat{y_{n-m}}$ in a neighborhood of the origin such that

$$
\widetilde{\omega}_{S}=\sum d \widehat{x}_{i} \wedge d \widehat{y}_{i}
$$

Finally, in the system of coordinates $\widetilde{p}_{1}, \hat{q}_{1}, \widehat{x}_{1}, \widehat{y}_{1}, \ldots, \widetilde{p}_{m}, \hat{q}_{m} \widehat{x}_{n-m}, \widehat{y}_{n-m}$, the symplectic form $\widetilde{\omega}$ has the standard form, the singular Lagrangian fibration is linear, and the free action of $\Gamma$ is also linear.

Thus we have shown that the original singular Lagrangian fibration near $O$ is symplectically equivalent to a linear model (direct if $\Gamma$ is trivial and twisted if $\Gamma$ is nontrivial).

As was mentioned in the introduction, the group of all linear automorphisms (i.e. linear moment map preserving symplectomorphisms) of the linear direct model of Williamson type $\left(k_{e}, k_{h}, k_{f}\right)$ is isomorphic to the following Abelian group:

$$
\mathbb{T}^{m} \times \mathbb{T}^{k_{e}} \times(\mathbb{R} \times \mathbb{Z} / 2 \mathbb{Z})^{k_{h}} \times\left(\mathbb{R} \times \mathbb{T}^{1}\right)^{k_{f}} .
$$

In particular, $\Gamma$ is necessarily a subgroup of $(\mathbb{Z} / 2 \mathbb{Z})^{k_{h}}$. (It comes from involutions of hyperbolic components, and it does not mix the components.) 
Remark. - In [15] and [14] Marle establishes a model for a Hamiltonian action of a compact Lie group in a neighborhood of an orbit. This result was obtained independently by Guillemin and Sternberg in [10]. In our construction a Hamiltonian action of an $m$-dimensional torus preserving the fibration determined by the moment map comes into the scene. In fact the linearization result we have just proved can be understood as a generalization of Guillemin-Sternberg-Marle theorem in the case the group considered is $\mathbb{T}^{m}$. It gives a linear model for a Hamiltonian action of a torus preserving an additional structure: a singular Lagrangian fibration.

In order to prove Theorem 2.1 it remains to consider the case when the compact symmetry group $G$ is nontrivial.

In the case there exists an action of a nontrivial group $G$ on $V / \Gamma$ we have an induced action of $G$ on $V$. The following theorem shows that this action can be linearized.

THEOREM 4.3. - Let $G$ be a compact Lie group preserving the system

$$
\left(D^{m} \times \mathbb{T}^{m} \times D^{2(n-m)}, \sum_{i=1}^{m} d p_{i} \wedge d q_{i}+\sum_{i=1}^{n-m} d x_{i} \wedge d y_{i}, \mathbf{F}\right)
$$

then there exists $\Phi_{G}$ a diffeomorphism defined in a tubular neighborhood of the orbit $L=\mathbb{T}^{m}$ which preserves the system

$$
\left(D^{m} \times \mathbb{T}^{m} \times D^{2(n-m)}, \sum_{i=1}^{m} d p_{i} \wedge d q_{i}+\sum_{i=1}^{n-m} d x_{i} \wedge d y_{i}, \mathbf{F}\right)
$$

and under which the action of $G$ becomes linear.

Proof. - After shrinking the original neighborhood if necessary, we may assume without loss of generality that we are considering a $G$-invariant neighborhood of $L$. First of all, let us express in local coordinates how the action looks like. We denote by $\rho$ the action of $G$. For convenience, we use the simplifying notation $p=\left(p_{1}, \ldots, p_{m}\right)$ and $(x, y)=\left(x_{1}, y_{1}, \ldots, x_{n-m}, y_{n-m}\right)$. Since $G$ preserves the system, in particular $\rho$ preserves $p$ and sends $\frac{\partial}{\partial q_{i}}$ to $\frac{\partial}{\partial q_{i}}$. After all these considerations, for each $h \in G$ the diffeomorphism $\rho(h)$ can be written as

$$
\rho(h)\left(p, q_{1}, \ldots, q_{m}, x, y\right)=\left(p, q_{1}+g_{1}^{h}(p, x, y), \ldots, q_{m}+g_{m}^{h}(p, x, y), \alpha^{h}(x, y, p)\right)
$$

where the functions $g_{i}^{h}$ and $\alpha^{h}$ are constrained by more conditions given by the preservation of the system. Before considering these constraints, it will be most convenient to simplify the expression of $\alpha^{h}$ first. This will be done using the local linearization theorem with parameters (Proposition 3.7).

In order to do that, we restrict our attention to the induced mapping,

$$
\bar{\rho}(h)(p, x, y)=\left(p, \alpha^{h}(p, x, y)\right)
$$

and we consider the family of diffeomorphisms $\bar{\rho}(h)_{p}: D^{2(n-m)} \rightarrow D^{2(n-m)}$ defined as follows

$$
\bar{\rho}(h)_{p}(x, y)=\alpha^{h}(p, x, y)
$$


We may look at $p=\left(p_{1}, \ldots, p_{m}\right)$ as parameters. For each $p$ the mapping $\bar{\rho}(h)_{p}(x, y)$ induces an action of $G$ on the disk $D^{2(n-m)}$ which preserves the induced system

$$
\left(D^{2(n-m)}, \sum_{i=1}^{n} d x_{i} \wedge d y_{i}, \mathbf{h}\right) .
$$

Observe that the preservation of the induced system implies, in particular, that the action fixes the origin.

According to Proposition 3.7 we can linearize the action $\bar{\rho}(h)_{p}$ in such a way that it taken to the parametric-free linear action $\bar{\rho}(h)_{0}^{(1)}$. We can extend trivially the diffeomorphism $\Phi_{p}$ in the disk provided by Proposition 3.7 to a diffeomorphism $\Psi$ in the whole neighborhood considered, simply by declaring, $\Psi\left(p, q_{1}, \ldots, q_{m}, x, y\right)=\left(p, q_{1}, \ldots, q_{m}, \Phi_{p}(x, y)\right)$. This diffeomorphism does not preserve (in general) the symplectic structure $\omega$, let $\omega_{1}=\Psi^{*}(\omega)$, we can apply Lemma 4.2 to $\omega$ and $\omega_{1}$. Consider the $\widetilde{g}_{i}$ given by Lemma 4.2 and define

$$
\widetilde{q}_{i}=q_{i}-\widetilde{g}_{i}
$$

Then after this change of coordinates $\omega_{1}$ is taken to $\omega$. For the sake of simplicity we will keep on using the notation $q_{i}$ for the new coordinates. After this linearization in the $(x, y)$-direction the initial expression of $\rho(h)$ looks like

$$
\rho(h)\left(p, q_{1}, \ldots, q_{m}, x, y\right)=\left(p, q_{1}+g_{1}^{h}(p, x, y), \ldots, q_{m}+g_{m}^{h}(p, x, y), \bar{\rho}(h)_{0}^{(1)}(x, y)\right) .
$$

Since the action preserves the symplectic form $\sum_{i=1}^{m} d p_{i} \wedge d q_{i}+\sum_{i=1}^{n-m} d x_{i} \wedge d y_{i}$ we conclude that the functions $g_{i}^{h}$ do not depend on $(x, y)$ and so far just depend on the parameters $\left(p_{1}, \ldots, p_{m}\right)$. In other words,

$$
\rho(h)\left(p, q_{1}, \ldots, q_{m}, x, y\right)=\left(p, q_{1}+g_{1}^{h}(p), \ldots, q_{m}+g_{m}^{h}(p), \bar{\rho}(h)_{0}^{(1)}(x, y)\right) .
$$

Observe that if we prove that these functions $g_{i}^{h}$ do not depend on $p$ then we will be done because then the induced action on $\mathbb{T}^{m}$ will be performed by translations. And, in all, the action will be linear.

Consider $\mathcal{H}=\{\rho(h), h \in G\}$, we are going to prove that this group is abelian.

We have to check that $\rho\left(h_{1}\right) \circ \rho\left(h_{2}\right)=\rho\left(h_{2}\right) \circ \rho\left(h_{1}\right)$.

We compute

$$
\begin{aligned}
& \rho\left(h_{1}\right) \circ \rho\left(h_{2}\right)\left(p, q_{1}, \ldots, q_{m}, x, y\right) \\
& \quad=\left(p, q_{1}+g_{1}^{h_{2}}(p)+g_{1}^{h_{1}}(p), \ldots, q_{m}+g_{m}^{h_{2}}(p)+g_{m}^{h_{1}}(p), \bar{\rho}\left(h_{1}\right)_{0}^{(1)} \circ \bar{\rho}\left(h_{2}\right)_{0}^{(1)}(x, y)\right) ;
\end{aligned}
$$

On the other hand

$$
\begin{aligned}
& \rho\left(h_{2}\right) \circ \rho\left(h_{1}\right)\left(p, q_{1}, \ldots, q_{m}, x, y\right) \\
& \quad\left(p, q_{1}+g_{1}^{h_{1}}(p)+g_{1}^{h_{2}}(p), \ldots, q_{m}+g_{m}^{h_{1}}(p)+g_{m}^{h_{2}}(p), \bar{\rho}\left(h_{2}\right)_{0}^{(1)} \circ \bar{\rho}\left(h_{1}\right)_{0}^{(1)}(x, y)\right) .
\end{aligned}
$$

Clearly, the first $2 m$ components coincide. As for the $2(n-m)$ last components, we can use the fact that $\mathcal{G}^{\prime}$, the group of linear transformations preserving the fibration and the symplectic form is abelian, as we pointed out in the introduction.

So far we know that the group $\mathcal{H}$ is abelian. It is also compact, therefore it is a direct product of a torus $\mathbb{T}^{r}$ with finite groups of type $\mathbb{Z} / n \mathbb{Z}$. We are going to check that for each $\rho(h) \in \mathcal{H}$ 
the functions $g_{i}^{h}$ do not depend on $p$. It is enough to check it for $\rho(h)$ in one of the components $\mathbb{Z} / n \mathbb{Z}$ and $\mathbb{T}^{r}$. So we distinguish two cases

- $\rho(h)$ belongs to $\mathbb{Z} / n \mathbb{Z}$. Then $\rho(h)^{n}=\mathrm{Id}$; this condition yields, $n g_{i}^{h}(p)=2 \pi m_{i}(p)$, $m_{i}(p) \in \mathbb{Z}$ for all $1 \leqslant i \leqslant m$. Since $m_{i}(p)$ is a continuous function taking values in $\mathbb{Z}$ it is a constant function $m_{i}$. Thus, $g_{i}^{h}(p)=\frac{2 \pi m_{i}}{n}$ and $g_{i}^{h}$ does not depend on $p$.

- $\rho(h)$ belongs to $\mathbb{T}^{r}$. We can consider a sequence $\rho\left(h_{n}\right)$ lying on the torus which belong to a finite group $\mathbb{Z} / k_{n} \mathbb{Z}$ and which converge to $\rho(h)$. For each of these points $\rho\left(h_{n}\right)$ we can apply the same reasoning as before to obtain $g_{i}^{h_{n}}(p)=\frac{2 \pi m_{i}}{k_{n}}$.

Now for each $n$, the diffeomorphism $\rho\left(h_{n}\right)$ does not depend on $p$; we may write this condition as

$$
\frac{\partial \rho\left(h_{n}\right)}{\partial p_{i}}=0, \quad 1 \leqslant i \leqslant m
$$

Now since the action is smooth we can take limits in this expression to obtain that

$$
\frac{\partial \rho(h)}{\partial p_{i}}=0, \quad 1 \leqslant i \leqslant m
$$

and finally $g_{i}^{h}(p)$ does not depend on $p$.

And this ends the proof of the theorem.

This linearizes the action of $G$ on $V$. After considering the quotient with the action of $\Gamma$ this theorem yields Theorem 2.1.

\section{Acknowledgements}

The authors wish to express their gratitude to the referee for the many suggestions done. We feel that these enlightening remarks have improved considerably the initial version of this paper. In fact, it was following the referee's suggestions that Theorem 3.2 was finally included in the paper.

The first author also wants to thank Carlos Currás-Bosch for his remarks and Ignasi Mundet $\mathrm{i}$ Riera for drawing her attention to the fact that the path used in the proof of Corollary 3.4 does also preserve any fibration with homogeneous component functions.

\section{Appendix A. Nonresonance versus nondegeneracy}

As pointed out by Ito [11,12] (see also [27]), in the real analytic case, the nondegeneracy condition explained in the introduction of this paper is essentially equivalent to the nonresonance condition. However, in the smooth case, this is no longer true: smooth integrable systems which are nonresonant at a singular point can be very degenerate at that point at the same time. In particular, we have:

PROPOSITION A.1. - Let $\gamma_{1}, \ldots, \gamma_{n}$ be any $n$-tuple of positive numbers which are linearly independent over $\mathbb{Z}, n \geqslant m+2, m \geqslant 0$. Then, there is a smooth integrable Hamiltonian function $H$ in a neighborhood of the (elliptic invariant) torus $\mathbb{T}_{0}^{m}=\mathbb{T}^{m} \times\{0\} \times\{0\}$ in the standard symplectic space $\left(\mathbb{T}^{m} \times \mathbb{R}^{m} \times \mathbb{R}^{2(n-m)}, \omega_{0}=\sum_{i=1}^{m} d p_{i} \wedge d q_{i}+\sum_{i=m+1}^{n} d x_{i} \wedge d y_{i}\right)$, such that $H=\frac{1}{\pi} \sum_{i=1}^{m} \gamma_{i} p_{i}+\sum_{i=m+1}^{n} \gamma_{i}\left(x_{i}^{2}+y_{i}^{2}\right)+$ higher order terms at $\mathbb{T}_{0}^{m}$, and such that $H$ does not admit a $C^{1}$-differentiable local Birkhoff normalization near $\mathbb{T}_{0}^{m}$ (i.e. the corresponding Lagrangian fibration cannot be linearized). Moreover, this integrable Hamiltonian function $H$ can be chosen so that it does not admit a nontrivial symplectic $\mathbb{S}^{1}$-symmetry near $\mathbb{T}_{0}^{m}$. 
In the above proposition, by integrability of $H$ we mean the existence of a smooth moment map $\left(F_{1}, \ldots, F_{n}\right)$ from a neighborhood of $\mathbb{T}_{0}^{m}=\mathbb{T}^{m} \times\{0\} \times\{0\}$ in $\mathbb{T}^{m} \times \mathbb{R}^{m} \times \mathbb{R}^{2(n-m)}$ to $\mathbb{R}^{n}$, with $F_{1}=H$, such that $\left\{F_{i}, F_{j}\right\}=0$, and $d F_{1} \wedge \cdots \wedge d F_{n} \neq 0$ almost everywhere. By "higher order terms" in $H$ we mean terms which are at least quadratic in variables $p_{i}$, or cubic in variables $p_{i}, x_{i}, y_{i}$. According to Sard's theorem (about the set of singular values) and Liouville's theorem, almost all common level sets of such a moment map are Liouville tori. The condition that $\gamma_{1}, \ldots, \gamma_{n}$ are independent over $\mathbb{Z}$ means that $\mathbb{T}^{m} \times\{0\} \times\{0\}$ is a nonresonant invariant elliptic torus of the Hamiltonian $H$. Recall that if there is a differentiable Birkhoff normal form, then (since we are in the elliptic case), the system also admits a Hamiltonian $\mathbb{T}^{n}$-symmetry near the elliptic singular orbit. Thus, if the system does not admit a nontrivial $\mathbb{S}^{1}$-symmetry near $\mathbb{T}_{0}^{m}$, then of course it cannot admit a differentiable Birkhoff normal form.

The proof of Proposition A.1 is inspired by what happens to generic perturbations of integrable systems: resonant tori that break up and give way to smaller-dimensional invariant tori, homoclinic orbits, diffusion, etc. Usually this breaking up of resonant tori leads to a chaotic behavior of the system, see, e.g., [21]. In order to prove Proposition A.1, we will construct an integrable perturbation of the quadratic Hamiltonian $\frac{1}{\pi} \sum_{i=1}^{m} \gamma_{i} p_{i}+\sum_{i=m+1}^{n} \gamma_{i}\left(x_{i}^{2}+y_{i}^{2}\right)+$ higher order terms at $\mathbb{T}_{0}^{m}$ in such a way that there are also invariant tori arbitrarily close to $\mathbb{T}_{0}^{m}$ that break up.

First let us consider the case with $m=0$ (i.e. a fixed point). Our construction of $H$ in this case consists of two steps.

Step 1. Creation of resonant regions.

Choose a smooth function $Q\left(I_{1}, \ldots, I_{n}\right)$ of $n$ variables $I_{1}, \ldots, I_{n}$ with the following properties:

a) $Q(0)=0$, and the linear part of $Q$ at 0 is $\sum \gamma_{i} I_{i}$.

b) There is a series of disjoint small open balls $U_{k}$ in $\mathbb{R}_{+}^{n}=\left\{\left(I_{1}, \ldots, I_{n}\right) \in \mathbb{R}^{n}, I_{1}>0\right.$, $\left.\ldots, I_{n}>0\right\}$, which tend to 0 (in Hausdorff topology) as $k \rightarrow \infty$, such that we have

$$
Q\left(I_{1}, \ldots, I_{n}\right)=\sum \gamma_{i}^{k} I_{i} \quad \forall\left(I_{1}, \ldots, I_{n}\right) \in U_{k}
$$

where $\gamma_{i}^{k}$ are rational numbers such that $\lim _{k \rightarrow \infty} \gamma_{i}^{k}=\gamma_{i}$.

Of course, such a function exists, and it can be chosen to be arbitrarily close to $\sum \gamma_{i}^{k} I_{i}$ in $C^{\infty}$-topology. Now put

$$
H_{1}=Q\left(x_{1}^{2}+y_{1}^{2}, \ldots, x_{n}^{2}+y_{n}^{2}\right) .
$$

Then for this integrable Hamiltonian function $H_{1}$, there are open regions $V_{k} \subset \mathbb{R}^{2 n}$ arbitrarily close to 0 in $\mathbb{R}^{2 n}$ which are filled by resonant tori in which the Hamiltonian flow of $H_{1}$ is periodic. These regions $V_{k}$ are preimages of the open sets $U_{k}$ chosen above under the moment $\operatorname{map}\left(I_{1}, \ldots, I_{n}\right)$.

Step 2. Creation of hyperbolic singularities.

We will modify $H_{1}$ inside each open subset $V_{k}$ by a $C^{\infty}$-small function which is flat on the boundary of $V_{k}$, in such a way that after the modification our Hamiltonian function remains integrable inside $V_{k}$ but admits an hyperbolic singularity there. Since $H_{1}$ has periodic flow in $V_{k}$ for each $k$, we can create a common model and then put it to each $V_{k}$ after necessary rescalings. The model can be done for a 2-dimensional system depending on $n-1$ parameters, and then take a direct product of it with $\mathbb{T}^{n-1}$. In the 2-dimensional case, it is obvious how to change a regular function on $D^{1} \times \mathbb{S}^{1}$ into a function with a hyperbolic singularity by a $C^{\infty}$-small perturbation. After the above modifications, we obtain a new smooth Hamiltonian function $\mathrm{H}_{2}$, which is $C^{\infty}$-close to $H_{1}$, which coincides with $H_{1}$ outside the union of $V_{k}$, and which is still 
smoothly integrable (though smooth first integrals for $H_{2}$ will necessarily be very degenerate at 0 ). Note that, by construction, the quadratic part of $H_{2}$ at 0 is $\sum_{i=1}^{n} \gamma_{i}\left(x_{i}^{2}+y_{i}^{2}\right)$.

Since $H_{2}$ has hyperbolic singularities arbitrarily near 0 , it cannot admit a differentiable Birkhoff normal form in a neighborhood of 0 , for simple topological reasons concerning the associated Liouville foliation. It is also easy to see that $H_{2}$ cannot admit an $\mathbb{S}^{1}$ symmetry near 0 : if there is a symplectic $\mathbb{S}^{1}$-action in a neighborhood of 0 which preserves $H_{2}$, then this action must also preserve the hyperbolic periodic orbits of $H_{2}$ in the resonant regions $V_{k}$. This, in turn, implies that there is a natural number $N$ such that $N \gamma_{i}^{k} \in \mathbb{Z}, \forall k, i$, which is impossible by our construction.

Let us now consider the case $m \geqslant 1$. For simplicity, we will assume that $m=1$. (The case $m>1$ is absolutely similar.) We will repeat the above two steps to create hyperbolic singularities, but with $n$ replaced by $n-1$ (recall that by hypothesis $n \geqslant m+2$ so $n-1 \geqslant 2$ ). The regions $V_{k}$ now lie in $R^{2 n-2}$, and $H_{2}=\sum_{i=2}^{n} \gamma_{i} I_{i}+\cdots$. By choosing our open sets $U_{k}$, we can assume that there is a function $F=F\left(x_{2}^{2}+y_{2}^{2}, \ldots, x_{n}^{2}+y_{n}^{2}\right)$ on $\mathbb{R}^{2 n-2}$, which is flat at 0 , and such that

$$
F=\frac{\pi}{k}\left(x_{n}^{2}+y_{n}^{2}\right) \quad \text { in } V_{k} .
$$

Denote by $\varphi$ the time-1 map of the Hamiltonian vector field $X_{F}$ of $F$ on $\mathbb{R}^{2 n-2}$. Observe that $\varphi$ is a symplectomorphism formally equivalent to the identity map at 0 , and that in each region $V_{k}$ the map $\varphi$ generates a nontrivial $\mathbb{Z} / k \mathbb{Z}$ symmetry (i.e. the $k$-times iteration of $\varphi$ in $V_{k}$ is the identity, and the lower iterations are not). Now we can construct $H_{2}$ in such a way that in each region $V_{k}$ it is also invariant under the $\mathbb{Z} / k \mathbb{Z}$ symmetry generated by $\varphi$. Then, since $H_{2}=H_{1}$ outside of the sets $V_{k}$, and $\left\{H_{1}, F\right\}=0$ by construction, the map $\varphi$ preserves $H_{2}$ everywhere in $\mathbb{R}^{2 n-2}$.

We now construct our symplectic manifold using suspension. More precisely, consider the free component-wise symplectic action of $\mathbb{Z}$ on $\mathbb{R}^{2} \times \mathbb{R}^{2 n-2}$, where the action of $\mathbb{Z}$ on $\mathbb{R}^{2 n-2}$ is generated by $\varphi$, and its action on $\mathbb{R}^{2}$ (with coordinates $(p, q)$ and symplectic form $d p \wedge d q$ ) is generated by the shift $(p, q) \mapsto(p, q+1)$. Then the suspension of $\varphi$ is the quotient of $\mathbb{R}^{2} \times \mathbb{R}^{2 n-2}$ by this $\mathbb{Z}$-action. Denote this quotient by $M$ and denote the projection by $\pi$. Let $\bar{V}_{k}$ be $\pi\left(\mathbb{R}^{2} \times V_{k}\right)$. Observe that the function $H_{2}$ is $\varphi$-invariant and both functions, $H_{2}$ and $p$, are invariant by the shift; therefore $H_{3}=\frac{\gamma_{1}}{\pi} p+H_{2}$ can be projected to $M$. We denote by $\bar{H}_{3}$ the projection of $H_{3}$. Since $H_{3}$ is integrable on $\mathbb{R}^{2} \times \mathbb{R}^{2 n-2}$ and the action defining the suspension is symplectic, the function $\bar{H}_{3}$ defines an integrable Hamiltonian system on $M$. We denote by $L_{k}$ an orbit of $X_{\bar{H}_{3}}$ through an hyperbolic singularity of $\bar{H}_{2}$ in the region $\bar{V}_{k}$ and denote by $L$ the orbit of $X_{\bar{H}_{3}}$ through the origin $O$. Observe that, by construction, the orbits $L$ and $L_{k}$ are circles. Let us see that there is no symplectic $\mathbb{S}^{1}$-action preserving $\bar{H}_{3}$ in a neighborhood of $L$. Assume there existed one, then the orbits of $X_{\bar{H}_{3}}$ would also be preserved by this action. Given an action of a Lie group $\phi: G \times M \rightarrow M$, we use the standard notation $G_{x}$ for the isotropy group at the point $x$. We are going to use the Slice theorem to reach a contradiction.

According to the slice theorem [20] for proper group actions there would exist a slice for the action through $x \in L$. Take $x=O$, we denote by $S$ the slice through the origin. From now on, we are going to consider a neighborhood of $L$ invariant by this $\mathbb{S}^{1}$-action. Then, there exists a $k_{0}$ such that for all $k \geqslant k_{0}$, the orbit $L_{k}$ is fully contained in this neighborhood. Since $S$ is a slice, the orbit $L_{k}$ is transverse to $S$ at each $p \in S$ and if $S \cap L_{k}$ is not empty then it consists of a finite number of points. Further, by construction, $S \cap L_{k}$ consists exactly of $k$ points $\left\{p_{1}, \ldots, p_{k}\right\}$ for $k \geqslant k_{1}$. Those points lie in an orbit for the action, therefore for each $p_{i}$ we can consider an element $g_{i} \in \mathbb{S}^{1}$ such that $\phi\left(g_{i}, p_{i}\right)=p_{1}$ and all the $g_{i}$ obtained in this way are different. Following [20], if $S$ is a slice though $x$ then for $p \in S$ and $g \in G$ the condition $\phi(g, p) \in S$ 
implies $g \in G_{x}$. Therefore all the elements $g_{i}$ are contained in $\mathbb{S}_{0}^{1}$, the isotropy group at the origin. By construction, $k$ tends to infinity as we are approaching the origin, and $\mathbb{S}_{0}^{1}$ is a compact group containing an infinity of elements, therefore $\mathbb{S}_{0}^{1}=\mathbb{S}^{1}$. This yields a contradiction because then the orbit through the origin would be reduced to a point. Therefore there exists no symplectic $\mathbb{S}^{1}$-action preserving $\bar{H}_{3}$.

This ends the proof of the proposition.

\section{REFERENCES}

[1] Bochner S., Compact groups of differentiable transformations, Ann. of Math. (2) 46 (1945) 372-381.

[2] ChAPERON M., Quelques outils de la théorie des actions différentiables, in: Third Schnepfenried Geometry Conference, vol. 1 (Schnepfenried, 1982), in: Astérisque, vols. 107-108, Soc. Math. France, Paris, 1983, pp. 259-275.

[3] Colin de Verdière Y., Vey J., Le lemme de Morse isochore, Topology 18 (1979) 283-293.

[4] COLIn DE VerdiÈre Y., Vu-NGOC S., Singular Bohr-Sommerfeld rules for 2D integrable systems, Ann. Scient. Éc. Norm. Sup. 36 (2) (2003) 1-55.

[5] CURRÁs-BOSCH C., unpublished note, 2001.

[6] CuRrás-Bosch C., Miranda E., Symplectic linearization of singular Lagrangian foliations in $M^{4}$, Diff. Geom. Appl. 18 (2) (2003) 195-205.

[7] Dufour J.-P., Molino P., Compactification d'actions de $\mathbb{R}^{n}$ et variables action-angle avec singularités, in: Symplectic Geometry, Groupoids, and Integrable Systems (Berkeley, CA, 1989), Springer, New York, 1991, pp. 151-167.

[8] Eliasson L.H., Hamiltonian systems with Poisson commuting integrals, Ph.D. Thesis, 1984.

[9] Eliasson L.H., Normal forms for Hamiltonian systems with Poisson commuting integrals - elliptic case, Comment. Math. Helv. 65 (1) (1990) 4-35.

[10] Guillemin V., Sternberg S., A normal form for the moment map, in: Sternberg S. (Ed.), Differential Geometric Methods in Mathematical Physics, Reidel, Dordrecht, 1984.

[11] Іто H., Convergence of Birkhoff normal forms for integrable systems, Comment. Math. Helv. 64 (3) (1989) 412-461.

[12] Іто H., Action-angle coordinates at singularities for analytic integrable systems, Math. Z. 206 (3) (1991) 363-407.

[13] Liouville J., Note sur l'intégration des équations différentielles de la dynamique, présentée au bureau des longitudes le 29 juin 1853, Journal de Mathématiques pures et appliquées 20 (1855) $137-138$.

[14] MARLE C.-M., Sous-variétés de rang constant d'une variété symplectique, in: Third Schnepfenried Geometry Conference, vol. 1 (Schnepfenried, 1982), in: Astérisque, vols. 107-108, Soc. Math. France, Paris, 1983, pp. 69-86.

[15] MARLE C.-M., Modèle d'action hamiltonienne d'un groupe de Lie sur une variété symplectique, Rend. Sem. Mat. Univ. Politec. Torino 43 (2) (1985) 227-251.

[16] MineuR H., Sur les systèmes mécaniques admettant $n$ intégrales premières uniformes et l'extension à ces systèmes de la méthode de quantification de Sommerfeld, C. R. Acad. Sci., Paris 200 (1935) 1571-1573.

[17] MinEUR H., Réduction des systèmes mécaniques à $n$ degré de liberté admettant $n$ intégrales premières uniformes en involution aux systèmes à variables séparées, J. Math. Pure Appl., IX Sér. 15 (1936) 385-389.

[18] MinEUR H., Sur les systèmes mécaniques dans lesquels figurent des paramètres fonctions du temps. Étude des systèmes admettant $n$ intégrales premières uniformes en involution. Extension à ces systèmes des conditions de quantification de Bohr-Sommerfeld, Journal de l'École Polytechnique, Série III, 143ème année (1937) 173-191, and 237-270.

[19] Miranda E., On symplectic linearization of singular Lagrangian foliations, Ph.D. Thesis, Universitat de Barcelona, 2003. 
[20] Palais R.S., On the existence of slices for actions of noncompact Lie groups, Ann. Math. 73 (2) (1961) 295-323.

[21] Sevryuk M.B., Invariant sets of degenerate Hamiltonian systems near equilibria, Regul. Chaotic Dyn. 3 (3) (1998) 82-92.

[22] VeY J., Sur certains systèmes dynamiques séparables, Amer. J. Math. 100 (3) (1978) 591-614.

[23] Weinstein A., Symplectic manifolds and their Lagrangian submanifolds, Advances in Math. 6 (1971) 329-346.

[24] Weinstein A., Lectures on Symplectic Submanifolds, Regional Conference Series in Mathematics, vol. 29, American Mathematical Society, Providence, RI, 1977.

[25] Zung NGUYen TIEN, Symplectic topology of integrable Hamiltonian systems, I: Arnold-Liouville with singularities, Compos. Math. 101 (2) (1996) 179-215.

[26] ZUNG NGUYen TIEN, A note on degenerate corank-one singularities of integrable Hamiltonian systems, Comment. Math. Helv. 75 (2) (2000) 271-283.

[27] ZUNG NGUYEN TIEN, Convergence versus integrability in Birkhoff normal form, math.DS/0104279, Ann. of Math. (2004), in press.

(Manuscrit reçu le 3 novembre 2003 ; accepté, après révision, le 15 octobre 2004.)

Eva MiRANDA

Departament d'Àlgebra i Geometria, Facultat de Matemàtiques, Universitat de Barcelona,

Gran Via de les Corts Catalanes 585, 08007 Barcelona, Espagne

E-mail: evamiranda@ub.edu

Nguyen Tien ZunG

Laboratoire Émile Picard, UMR 5580 CNRS, UFR MIG,

Université Toulouse III,

31062 Toulouse Cedex, France

E-mail: tienzung@picard.ups-tlse.fr 